\title{
One Step Hydrothermal Synthesis of Ni-MoS2-RGO Bifunctional Electrocatalysts for HER and OER
}

\author{
Min Zhao ${ }^{1,2}$, Gaoliang Zhou ${ }^{l}$, Xiaoman Liu ${ }^{l}$, Xinyi Shen ${ }^{l}$, Jianguo Lv ${ }^{1,2, *}$, Changjuan Hu ${ }^{l}$, Ying \\ Wang $^{1}$, Wei Tan ${ }^{1}$, Shengjie Sun ${ }^{1}$, Yuxuan Ma ${ }^{1}$, Congrong Wang ${ }^{1}$, \\ Jin Yang ${ }^{1}$, Miao Zhang, 3,, Gang He ${ }^{3, *}$, Lei Yang ${ }^{4}$ \\ ${ }^{1}$ School of Physics and Materials Engineering, Hefei Normal University, Hefei 230601, China \\ ${ }^{2}$ Key Laboratory for Photoelectric Detection Science and Technology of Education Department of \\ Anhui Province, Hefei Normal University, Hefei 230601, China \\ ${ }^{3}$ School of Physics and Material Science, Anhui University, Hefei 230039, China \\ ${ }^{4}$ Department of Chemistry and Materials Engineering, Hefei University, Hefei 230601, China \\ *E-mail: 1vjg1@163.com, zhmiao@ahu.edu.cn, hegang@ahu.edu.cn
}

doi: $10.20964 / 2021.03 .46$

Received: 11 November 2020 / Accepted: 30 December 2020 / Published: 31 January 2021

Pure $\mathrm{MoS}_{2}$ and Ni-MoS $2-\mathrm{RGO}$ were prepared by a one-step hydrothermal approach. The result indicated that intensity of XRD diffraction peaks for $\mathrm{MoS}_{2}$ increase slightly as with the increase of RGO content. Mesopores of the $\mathrm{MoS}_{2}, \mathrm{Ni}-\mathrm{MoS}_{2} / \mathrm{RGO}-10$ and Ni-MoS$/$ RGO-15 are mainly distributed in the pore diameter rage of 2-50 nm. The addition of RGO increase the layer numbers of $\mathrm{MoS}_{2}$ in Ni-MoS$/ 2 \mathrm{RGO}$. Two Raman characteristic peaks at about 378 and $400 \mathrm{~cm}^{-1}$ according to $A_{1 \mathrm{~g}}$ and $\mathrm{E}^{1}{ }_{2 \mathrm{~g}}$ modes have been observed in the $\mathrm{MoS}_{2}$ and Ni-MoS$/$ RGO. The eletrocatalytic test results show that the Ni-MoS$/ \mathrm{RGO}_{2}$ 5 has the lowest overpotentials of $349 \mathrm{mV}$ at a current density of $10 \mathrm{~mA} \mathrm{~cm}^{-2}$ for OER and the Ni$\mathrm{MoS}_{2} / \mathrm{RGO}-10$ has the lowest overpotentials of $398 \mathrm{mV}$ at a current density of $10 \mathrm{~mA} \mathrm{~cm}{ }^{-2}$ for HER. Moderate Ni doping and RGO composites can effectively improve the intrinsic conductivity, the hydrogen adsorption free energy for HER, as well as providing more active sites for HER and OER.

Keyword: Ni-MoS2-RGO; Oxygen evolution reaction; electrocatalysts; Hydrothermal method

\section{$\underline{\text { FULL TEXT }}$}

(C) 2021 The Authors. Published by ESG (www.electrochemsci.org). This article is an open access article distributed under the terms and conditions of the Creative Commons Attribution license (http://creativecommons.org/licenses/by/4.0/). 